\title{
BMJ Open Dancing to improve balance control, cognitive-motor functions and quality of life after stroke: a study protocol for a randomised controlled trial
}

\author{
Emmanuel Morice, ${ }^{1}$ Julien Moncharmont, ${ }^{1}$ Clémentine Jenny, ${ }^{1}$ \\ Anne-Violette Bruyneel (i) ${ }^{2}$
}

To cite: Morice $\mathrm{E}$,

Moncharmont J, Jenny C, et al. Dancing to improve balance control, cognitivemotor functions and quality of life after stroke: a study protocol for a randomised controlled trial. BMJ Open 2020;10:e037039. doi:10.1136/ bmjopen-2020-037039

- Prepublication history and additional materials for this paper is available online. To view these files, please visit the journal online (http://dx.doi. org/10.1136/bmjopen-2020037039).

Received 16 January 2020 Revised 19 August 2020 Accepted 25 August 2020

D) Check for updates

(c) Author(s) (or their employer(s)) 2020. Re-use permitted under CC BY-NC. No commercial re-use. See rights and permissions. Published by BMJ.

${ }^{1}$ Neurorehabilitation, Institution de Lavigny, Lavigny, Vaud, Switzerland

${ }^{2}$ Physiotherapy Department, Geneva School of Health Sciences, HES-SO University of Applied Sciences and Arts Western Switzerland, Geneva, Switzerland

\section{Correspondence to}

Professor Anne-Violette Bruyneel;

anne-violette.bruyneel@hesge. ch

\section{ABSTRACT}

Introduction Dance is an intrinsically motivating activity that includes social interaction, stimulation through music, the pleasure of moving despite pathology-induced motor limitations, and it also has good perceived benefits among participants. Feeling pleasure while moving is essential to finding the motivation to engage in a rehabilitation programme. It is, therefore, urgent to provide persons in a poststroke situation with motivating physical activity opportunities. Very few studies have examined dance in a stroke context, while it is highly adapted and effective for other chronic conditions.

Our primary objective is to assess the effects of dance programme on patients' balance control after stroke. Our secondary objective is to investigate the effects of dance on cognitive function, strength, coordination, functional status, balance confidence, quality of life, motivation and adherence. Our hypothesis is that dance increases balance and motor capacities, and improves poststroke quality of life, adherence and motivation.

Methods and analysis Forty-eight subjects with stroke in subacute phase will be randomised into two groups: (1) intervention (dance and standard rehabilitation) and (2) control (standard rehabilitation). Before intervention, stroke severity, cognitive abilities and motor capacities will be assessed. Two baseline tests will be planned to evaluate the stability of individuals. Participants will attend a weekly 60-min dance class for 6 weeks. Cognitive and motor functions (balance, lower-limbs strength, coordination and motor level), quality of life (Stroke-Specific Quality of Life Scale) will be measured at weeks 4 and 6 in both groups. Participant satisfaction with regard to dance will be tested, as well as adherence and adverse effects.

Ethics and dissemination Ethics approval has been granted by the Swiss Ethics Committee of the CER Vaud (2019-01467). Outcomes will be disseminated through publication in peer-reviewed journals and presentations at conferences.

Trial registration number NCT04120467.

\section{BACKGROUND AND RATIONALE}

Dance seems to be a particularly relevant activity for patients with stroke. It could be an effective way to improve recovery, wellbeing and tackle sedentariness-related risks.
Strengths and limitations of this study

- This is a pioneering randomised control trial to assess the effects of dance programme in individuals after stroke in subacute phase.

- The primary outcome measure is the balance performance (Mini-BESTest) and assessed by a rater masked to group allocation.

- Since the participants will be in the subacute phase, the stability will be verified by a double-baseline assessment.

- Fidelity to dance practice and usual rehabilitation will be highly monitored.

- It is difficult to perform a double-blind study since participants know what exercise is being performed (dance+usual rehabilitation vs usual rehabilitation).

Joy, ${ }^{1}$ satisfaction ${ }^{2}$ and the benefits of dancewhich are genuinely perceived by the participants-increase intrinsic motivation, which in turn fosters adherence to physical activity. ${ }^{3}$ Moreover, high adaptability of dance makes it an easy practice for patients, for it can cover very broad motor and cognitive aspects. This point is exceedingly important because tasks that are too difficult or too easy will lead to lower levels of motivation, reduced motor learning and adherence (extent to which patients' behaviours are in accordance with the recommendations of healthcare). ${ }^{4}$ All these elements may explain the success of dance programmes applied to patients with chronic diseases. ${ }^{5}$

Stroke prevalence remains high (15 254 cases in 2016 according to Switzerland's Federal Statistical Office) and is also the country's first cause of disability. ${ }^{6}$ Following a brain lesion-due to ischaemic or haemorrhagic disturbances of cerebral blood circulationvarious deficits will impact patients' functional abilities, autonomy and health costs. ${ }^{7}$ Motor recovery is, therefore, an essential part 
of the therapeutic strategy in order to optimise patients' capacities and ensure that they age in the best conditions possible despite their disability. ${ }^{8}$ Stroke mainly induces cognitive and motor disturbances (balance, gait, muscle tone, muscle power, coordination, proprioception) that must be included into the rehabilitation process. ${ }^{9}$ Thus, the recovery assessment should primarily focus on these aspects.

A Cochrane review showed that rehabilitation should be considered effective in promoting motor recovery, reducing cognitive and motor deficits and improving independence. $^{8}$ Nevertheless, the exercises' modalities are unclear. Aquatherapy, ${ }^{10}$ split-bell training, ${ }^{11}$ aerobic training ${ }^{12}$ and cycling ${ }^{13}$ have shown interesting effects on rehabilitation after stroke, and also show a willingness to diversify the exercises. Recent research on neuroplasticity mechanisms after stroke has shown the importance of treatment optimisation-such as physical exercises including aerobic modalities and an enriched environment that simultaneously integrates cognitive, motor and social aspects. ${ }^{14}{ }^{15}$ Green et al highlighted that natural training is better than specific exercises. ${ }^{4}$ Natural activities are characterised by complex tasks, with self-feedback, integrating the whole body and diversified in terms of attentional, cognitive, motor and relational requirements. Thus, these tasks promote generalised learning, not only leading to a more energy-efficient movement strategy throughout the target task, but also to applying this learning to new tasks and contexts. ${ }^{4}$ These natural activities also have the advantage of developing a motor and cognitive dual-task training that was more effective in improving balance and gait abilities than single-task training. ${ }^{16}$ The physical activities that seem to be the most effective for recovery are the ones that can be adapted to the patient's level, offer varied and complex constraints for the whole body, induce motivation, have a social component and provide self-feedback. ${ }^{47}$

There is a growing interest in the practice of art as a means of helping patients, for such activities leave the usual healthcare framework and effectively include physical, mental and social well-being. ${ }^{18}$ Indeed, singing shows improvements in relaxation, breathing, posture, social interaction and emotional strain. ${ }^{19}$ However, music shows limited physical effects, which is why some authors studied circus and dance-which involve balance, endurance, mobility, muscular strength and coordination. ${ }^{2021}$

A previous review highlighted the benefits of dance in terms of physical, mental and social well-being in 51 articles about chronic pathologies. ${ }^{22}$ Motor function, balance and symptoms are the most studied factors in their relation to dance. ${ }^{22}{ }^{23}$ No adverse effect was reported, and patient adherence seems excellent. These positive effects were observed for neuromusculoskeletal, psychiatric, cardiovascular, metabolic and oncological pathologies. ${ }^{22}$ Regarding Parkinson's disease, two reviews showed improvements in symptoms and speed of the Timed Up and Go test when compared with other exercises. ${ }^{524}$ Patterson et al highlighted that dance classes are suitable for adults with neurological conditions and improve gait and balance parameters, but there are few studies on stroke. ${ }^{25}$ Only two studies on subjects with stroke, with no control group, showed the feasibility of a dance programme, a high satisfaction rate of participants, some benefits regarding balance and no adverse effect. ${ }^{20}{ }^{26}$ Dance seems particularly adapted to the multidimensional impairments of patients with stroke, for example, cognitive, motor, balance, quality of life and social impairments. ${ }^{9}$ Participants seem to rediscover the pleasure of moving-despite their disabilities-and enjoy a strong social bond that favours inclusion and adherence. During the subacute phase, aerobic physical activity and the enriched environment provided by dance programmes should enhance recovery while working specifically on balance, coordination, strength, mobility and cognitive aspects. Moreover, O'Connell et $a l^{18}$ highlighted that of all artistic activities, dance was the most common before stroke, and that patients express their desire to continue this activity inside the hospital. Given all these aspects, dance should be a particularly well-adapted and interesting way of optimising patient recovery after stroke.

\section{Objectives}

Primary objective

This study is a randomised controlled trial (RCT), which aims to investigate the effects of dance programmeassociated with conventional treatments-on the balance control of subjects with stroke (subacute phase), compared with conventional treatment alone. Our hypothesis is that a 6-week dance programme associated with conventional rehabilitation will improve balance control recovery when compared with conventional rehabilitation alone.

\section{Secondary objectives}

Our secondary objective is to assess the effects of dance programme combined with conventional rehabilitation on cognitive function, lower-limb strength, coordination, functional status, balance confidence and quality of life. The motivation to engage in physical activity, satisfaction and adherence will also be tested. Our hypothesis is that the practice of dance during 6 weeks will induce strong satisfaction and adherence, and increase quality of life and motivation to engage in physical activity-compared with conventional rehabilitation alone. The adverse effects of dance in subjects with poststroke hemiparesis in the subacute phase will be assessed.

\section{METHODS AND ANALYSIS}

\section{Trial design and setting}

The study protocol is approved by the Swiss Ethical Committee (CER Vaud 2019-01467). A flow chart of the study process is shown in figure 1 .

The study protocol conforms to the Standard Protocol Items: Standard Protocol Items for Randomised Trials. 


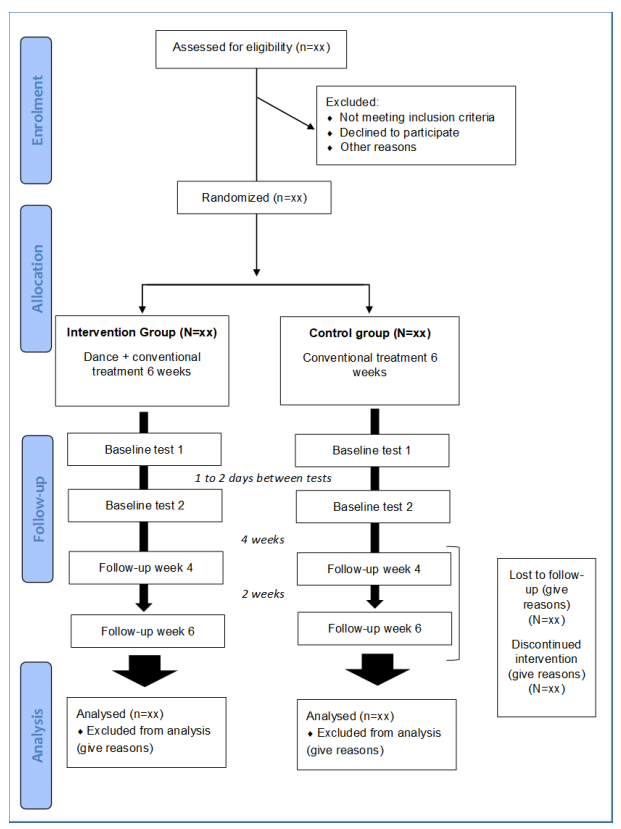

Figure 1 Flow chart of the study protocol.

\section{Patient and public involvement statement}

This work does not feature patient and public involvement in clinical research. Instead, we chose to observe dance classes with patients with neurological conditions and we included neurorehabilitation clinicians in the conception of this research. Thus, it is a coproduction between researchers and clinicians.

\section{Participants}

\section{Eligibility criteria}

Individuals will be eligible for inclusion if the first occurrence of a stroke is less than 3 months old (subacute phase) with medical stability (eg, multiple strokes, cardiorespiratory impairments), if they are 45-80 years old, if they are able to understand the instructions (Mini-Mental State Examination $>15$ ), to maintain a sitting position during $30 \mathrm{~s}$ and to endure $60 \mathrm{~min}$ of moderate physical activity. Participants will be excluded if they present severe hearing loss, pre-existing conditions that affect gait and balance, or major cognitive disturbances. This study will not include vulnerable subjects according to the criteria of the Swiss Human Research law.

\section{Recruitment procedure}

Two physiotherapists from the Institution de Lavigny (Lavigny, Vaud, Switzerland) neurorehabilitation centre and the study investigator will be responsible for the recruitment. All individuals will be consecutively recruited. During their hospitalisation, patients will be identified as potentially eligible based on data from their clinical file (date of birth, date of stroke, stage of stroke, presence of hemiparesis, clinical data). Diagnosis for poststroke hemiparesis should be systematically established by a neurologist, based on medical imaging and clinical examination. Oral and written information about the study will be provided to each patient by a member of the research team. Patients are recommended to take at least 24 hours to consider and discuss participation with a relative before deciding on participating in the study.

\section{Randomisation}

Participants who meet the eligibility criteria and sign the informed consent form will have baseline data collected prior to the randomisation procedure. To ensure allocation concealment, randomisation to groups (dance or control group) will be undertaken by an independent investigator not involved in the experiment, using a computer-generated randomisation tool. We will choose a randomisation method using permuted-block randomisation (ie, random block sizes of 4 and 6), with a 1:1 allocation ratio, ensuring complete randomness of subject assignment to each randomised group. The random assignment sequence will be computer generated and stored electronically by the independent investigator. The dance teacher will remain unaware of the allocation until participants have been fully accepted. The dance teacher will be informed of the allocation by email (allocation concealment will not be possible for this person at this point)

\section{Blinding}

Researchers responsible for the interventions will be blinded to the participants' initial assessments, and the researcher responsible for the evaluations will be unaware of each participant's group. Moreover, data collected during the evaluations will not be revealed to the researchers responsible for the interventions, and participants will be instructed not to disclose their experience or information related to the intervention. Finally, the researcher responsible for the statistical analysis will be blinded.

\section{Initial clinical information}

In order to identify the demographic and clinical characteristics of the participants, information regarding their age, sex, type of stroke, affected brain areas and medical history will be collected from their medical charts. Clinical tests will then be conducted to determine the motor level of the subjects' lower limbs (Chedoke Mc Master Assessment ${ }^{27}$ ), the spontaneous and rapid $10 \mathrm{~m}$ walking speed, ${ }^{28}$ the functional mobility (Timed Up and Go), the trunk balance (Trunk Control Test), the hemineglect (Bells test) and the dance experience. ${ }^{20}$

\section{Interventions}

\section{Dance group}

One group of 2-8 participants will attend a 60 min dance class weekly over a 6 -week period during their rehabilitation at the Lavigny Hospital in a gym. This one-class-perweek frequency is justified by previous studies ${ }^{2930}$ and the availability of the centre's resources. A physiotherapistdance teacher will lead the classes, supported by volunteers (physiotherapists or physical activity specialists). The number of volunteers (1-2) may change according to the needs of the participants. The group can include 
Table 1 Example of the structure of a typical dance class

\begin{tabular}{|c|c|c|}
\hline Elements & Duration (minutes) & Description \\
\hline 1. Warm-up & 10 & $\begin{array}{l}\text { In a seated position, warm-up including active range of motion of all } \\
\text { joints (from neck to toes), slow passive stretching of the most affected } \\
\text { legs and muscular awakening of the different segments and balance } \\
\text { exercises. Basic dance step to increase heart rate. }\end{array}$ \\
\hline 2. Technical exercices & 10 & $\begin{array}{l}\text { Mirror work. Learning a sequence of movements (basic technical dance } \\
\text { step) that the participants will have to reproduce. } \\
\text { Standing and/or chair choreography will be focused on strength, balance } \\
\text { and coordination. }\end{array}$ \\
\hline 3. Improvisation & 15 & $\begin{array}{l}\text { Creative and relational work. } \\
\text { Participants will have to interact with one or more partners. } \\
\text { Participant were instructed to move freely according to some } \\
\text { directions (large and big movements, unilateral or bilateral movements, } \\
\text { environmental constraints). In turn, they must either guide and be } \\
\text { followed or follow the sequence proposed by another dancer. }\end{array}$ \\
\hline 5. Cool down/feedback time & 10 & $\begin{array}{l}\text { Breathing exercises. Phase of return to calm (slow range of motion of all } \\
\text { joints) and exchanges on the session. }\end{array}$ \\
\hline
\end{tabular}

other participants with other neurological disabilities to complete the number of participants, however they will not be included in this study.

All dance classes will be accompanied by music. Each class will be divided into five steps (table 1). ${ }^{20}{ }^{26}$ Dance exercises will be targeting flexibility, balance, endurance, upper and lower limbs functions, interaction between dancers, perception (visual imagery and inclusion of the affected side) and memory. The dance style will be a combination of contemporary and social dance (eg, tango), with a focus on body/space interaction and the interaction with other persons in the same space. Using choreography or short routine, the class will involve repeating dance moves-which fosters memorisationwith also the additional challenge of remembering a sequence of moves.

Each dance step will be demonstrated by the dance teacher and shall be the same for all participants. However, given the great variability of each participant's functional ability, the complexity and intensity of dance will progress according to each participant's capacity-for it to be an appropriate challenge. All the dance moves will have to be possible sitting or standing.

In parallel, all participants will have the conventional poststroke follow-up care, which respects the Institution de Lavigny Institution's approach.

\section{Control group}

The control group will also receive the same 6-week follow-up, only without the dance class. The conventional treatment includes 45-60 min of physiotherapy per day by the Lavigny centre therapy team. This therapy consists of sensory stimulation, motor activation, strengthening, coordination, balance, physical training according to the objectives set up from the assessment. The physical therapists will choose the adapted exercises according to the rehabilitation process' main objective. In addition, group sessions are scheduled 2-3 times per week depending on the patient's abilities. The group sessions of $2-8$ patients can be 'walking groups', 'fitness training groups', 'assistive biking groups', 'cooking groups' and 'speak training groups'. They will be led by an interdisciplinary team of two different therapists. Every day, patients will also have an occupational therapy session which will include training in daily activities. From 3 to 5 times a week, patients will have neuropsychology and speech therapy sessions which will include cognitive function training through paper or computer exercises.

\section{Outcomes measures}

The psychometric qualities of the tests used are presented in table 2 .

\section{Primary outcomes}

The primary outcome is balance control recovery, measured through change assessment (compared with baseline) in cognitive-motor functions for both groups (dance vs control).

Balance recovery will be measured using the Balance Evaluation System test (mini-BESTest). The test has a maximum score of 28 points from 14 items that are each scored from 0 to 2. '0' indicates the lowest level of function and ' 2 ' the highest level of function. ${ }^{31}$

\section{Secondary outcomes}

Cognitive function will be measured using the Montreal Cognitive Assessment (MoCA) scale. ${ }^{32}$ This tool is better than the Mini-Mental State Evaluation with less of a ceiling 
effect and higher internal reliability; it is a stronger functional status indicator. ${ }^{32}$ There are 12 items in the cognitive domain: memory is tested through a short-term memory recall task (five points); visuospatial ability is tested using a clock-drawing test (three points) and a three-dimensional cube copy (one point); executive function is tested using a trail-making test (one point), a phonemic fluency task (one point), and a two-item verbal abstraction task (two points); attention, concentration and working memory are tested using an attention task (one point), a serial subtraction task (three points) and digits tasks (one point each); language is tested using a three-item confrontation naming task with animals (three points) and repetition of two complex sentences (two points); orientation in time and place is also tested (six points). The minimum score is 0 and the maximum score is 30 . Higher scores indicate better cognition (normal score:>26/30).

Strength will be measured using knee extensors' muscle with a hand-held dynamometer-in Newton (N). Indeed, this muscle group represents very well the strength of other muscles of the lower limb in stroke. ${ }^{33}$ Participants will be in a sitting position with their feet free and a $90^{\circ}$ knee flexion. The dynamometer will be placed on the distal part of the leg. ${ }^{345}$ Participants will apply maximum pressure on the dynamometer for $5 \mathrm{~s}$. Three tests will be carried out for the non-paretic and paretic sides.

Coordination recovery will be tested using the Lower Extremity Motor Coordination (LEMOCOT) test. ${ }^{36} 37$ Subjects will be sat on an adjustable chair with their feet resting flat on thin rigid foam, heels on the proximal target, and with knees and hips at $90^{\circ}$ flexion. Then, after a first trial, they will be instructed to alternately touch the proximal and distal targets, placed $30 \mathrm{~cm}$ apart, using their big toe, for $20 \mathrm{~s}$, as fast as possible, without trading accuracy for speed. The number of touched targets will be counted and registered for analysis.

Motor level will be assessed using a Functional Independence Measure Instrument (FIM) ${ }^{38}$ The scale contains 18 items, 13 of which concern physical domains and five of which are cognition related. Based on the level of independence, each item is scored from 1 to 7 , where 1 indicates total dependence and 7 represents complete independence. Possible scores range from 18 to 126. Higher values represent a better outcome.

Balance confidence will be measured using the Activities-Specific Balance Confidence scale (ABCscale). ${ }^{39}$ The ABC-scale is a self-reported questionnaire providing information on balance confidence in the performance of 16 different daily activities. The questionnaire contains 16 items scored on a range from $0 \%$ to $100 \%$ (0: no confidence and 100: full confidence). A total score of $\leq 67$ indicates an increased risk of fall. Standing balance will be tested in standing in single task and dualtask (with phone text task) with an inertial sensor for extracted displacement in anteroposterior and mediolateral directions. ${ }^{40} 41$

The quality of life of persons with chronic stroke will be measured using the Stroke-specific quality of 
life scale (SSQoL). The SS-QOL contains 12 subscales with a total of 49 items. The scoring of the SS-QOL will cover the previous week and is rated on a 5-point Likert scale. Response options are scored as 5 ('no help needed/no trouble at all/strongly disagree'), 4 ('a little help/a little trouble/moderately disagree'), 3 ('some help/some trouble/neither agree or disagree'), 2 ('a lot of help/a lot of trouble/moderately agree'), and 1 ('total help/could not do it at all/strongly agree'). The SS-QOL provides domain scores and a summary score, with higher scores indicating better function. The minimum score is 12 and the maximum score is 245. This questionnaire is adapted to the subacute phase and is validated in French. ${ }^{42}$

The motivation scale towards health-oriented physical activity (MS-PA) contains a total of 18 items. $^{43}$ Scoring is rated on a 7-point scale ( $1=$ 'strongly disagree' and $7=$ 'strongly agree'). The minimum score $=18$ and maximum score $=126$. Higher values represent less motivation.

The adverse effects of the dance programme will be assessed. Pain and fatigue will be measured using the Numeric Rating Scale (NRS) before and after each dance class. The 11-point numeric scale ranges from ' 0 ' (eg, 'no pain' or 'no fatigue') to '10' (eg, 'pain as bad as you can imagine' or 'worst pain imaginable' or 'worst fatigue imaginable'). Higher scores indicating greater pain or fatigue intensity.

Participant satisfaction will be assessed with an exit survey adapted from a previous study report that investigated dance for persons with chronic stroke. ${ }^{20}$ The survey asked participants to rate nine statements about the dance programme using a 5-point scale (where $1=$ 'strongly disagree' and $5=$ 'strongly agree'). Higher values represent better satisfaction. Adherence will be evaluated by the number of sessions actually attended compared with the number of dance classes and conventional interventions planned.

\section{Data collection and management}

Data will be collected twice (at baseline 1 and baseline 2) to evaluate the stability of the studied parameters. If the results of baseline 1 and baseline 2 are different, we will use baseline 2's and treat baseline 1 as a run-in test. If the results appear similar, we will use the average. The follow-up will include two assessments with data collection: at 4 weeks of intervention and then at 6 weeks to evaluate the changes from baseline (figure 1). The first follow-up will be an intermediate evaluation justified by the evolution of the patients in the subacute phase ${ }^{44}$ and because studies have shown the positive effects of dance after 4 weeks. ${ }^{26}$ Given that the decreased hospitalisation time in the hospital strategies, it will be interesting to assess the effectiveness after 1 month. The 6-week follow-up is in line with the usual hospital stay of patients with stroke at the rehabilitation centre. If the participant is discharged before the end of the follow-up, they will have to go back to the centre for the dance classes. During the entire process, each adverse event will be registered and reported to the medical staff.

In order to promote data quality, examiners will be physiotherapists/researchers with experience in neurology. All tests were chosen according to our objectives and their psychometric qualities. Measurements will be made at the neurorehabilitation centre by project workers specifically trained in the test protocol in order to standardise the data collection procedure.

The following measures will be taken for data quality: double data entry, weekly data back-up and training of those involved in the study in all important aspects of the study.

Data management has been approved by the Swiss ethics committees. Identifiable information data will be kept separate from the main dataset and will not be shared. All personal data will be stored securely in order to protect confidentiality before, during and after the trial. Data entry and coding of the de-identified data will be conducted by trained staff.

\section{External monitoring}

As this study presents no more risks than the usual management of patients after stroke, no external monitoring will be necessary. However, an internal monitoring is planned. A professor, who is not part of the project, will ensure that the rights and welfare of the participants are respected, that the data reported is accurate and verifiable from the source documents, and that the trial is conducted and documented in accordance with the protocol, procedures and applicable regulations.

\section{Withdrawal and discontinuation}

Participants will be free to withdraw from the trial at any point. The criteria for subject withdrawal will be: a deterioration of medical stability; a misunderstanding of the tests or the activity not delivering satisfying results; pain during tests and/or dance classes (NRS $>5$ ); withdrawal of informed consent; the procedure or routine will be stopped for safety reasons; behavioural disorders during dance classes. Withdrawal motivations will be reported for both groups. In case of withdrawal, a final assessment will be conducted for safety. Since recruitment is consecutive, it is possible to extend the recruitment phase to complete the trial.

In case of withdrawal, all data obtained will be made anonymous by replacing-in all documents-the sources of identification by the subject number. No further identification will then be possible.

\section{Statistical analysis}

\section{Sample size calculation}

The sample size was calculated from the Mini-BESTest results in patients with subacute stroke. ${ }^{45}$ This test has the highest psychometric characteristics with a low floor/ceiling effect and good sensitivity to change for 
subjects after stroke. In addition, in the study of Patterson et $a l^{20}$ on dance and stroke, this test was used as an outcome indicator and showed that it correctly identified patient changes. A 3.5/28-point difference in change is considered clinically relevant for persons with stroke in subacute phase. ${ }^{45}$ To detect this difference, 21 participants per group are needed with an SD of 3.9/28, power at $80 \%$ and a significant threshold of $\mathrm{p}<0.05$ in two-tailed test. To deal with possible withdrawals, we will include 24 subjects per group.

\section{Data treatment and statistical analysis}

The statistical analysis will be performed blinded to group allocation, using the software SPSS (SPSS inc. Chicago, IL, USA, V.22.0, 2020).

The first data treatment will include normalisation with body weight for quadriceps strength and data extraction for balance measures. When different trials are conducted, the different trials' mean calculation for each condition will be calculated.

Absolute and relative frequencies will be determined for categorical variables. Quantitative data will be analysed with the Shapiro-Wilk test in order to confirm their distribution and to determine the correct statistical tests. Means and SD will be calculated for normally distributed variables. The numerical median and range will be calculated for non-normally distributed variables. The calculations will be performed for each data acquisition time point and each group.

The Mini-BESTest score variable will be treated as primary outcome for the 6 weeks measure. If the variable presents a normal distribution, a two-way repeated measures analysis of variance (ANOVA) will be used to determine how this dance intervention impacts on balance control. The ANOVA incorporated the groups (dance vs control), time (baseline, 4 weeks and 6 weeks) and the group $\mathrm{x}$ interaction. Primary analysis will assess if mini-BESTest change at 6 weeks compared with baseline is different between intervention and control groups. We will do post hoc comparison between 6 weeks and 4 weeks then between 4 weeks and baseline as secondary analyses.

Multivariate ANOVA will also be performed for each secondary variable of interest, including the MoCa score, knee extension strength, LEMOCOT, FIM score, SSQoL, MS-PA, ABC-Balance confidence, ML and AP displacements for standing balance, pain, fatigue, satisfaction with dance class, adherence and adverse effects. If there are baseline differences between the groups, analysis of covariance will be used to eliminate the influence of extraneous factors.

If the distribution is not normal, the non-parametric Mann Whitney U test will be used to compare the groups in each time evaluation, and the Wilcoxon signed-rank test to compare evaluation time for each group baseline, 4 weeks and 6 Weeks. In case of qualitative data, the $\chi^{2}$ test will be applied.

A threshold value of $p<0.05$ (two-tailed test) will be adopted to rule out non-significant differences.
ETHICS AND DISSEMINATION

\section{Recruitment and consent}

Prospective, written consent (online supplemental file) will be obtained from all participants.

\section{Data collection, storage and access}

Data will be deidentified and entered into a secure folder and electronic database. Only the investigators and a statistician will have access to the final dataset.

\section{Dissemination strategy}

Outcomes will be disseminated through publication in peer-reviewed journals and presentations at international conferences.

\section{DISCUSSION}

There is a growing interest in the use of dance as a therapeutic tool for people with chronic diseases. ${ }^{22}$ Previous reviews showed that dance is an activity that involves the whole body in movements with variable constraints, which seems to have positive impacts on physical, motor, cognitive and relational disorders. ${ }^{523-25} 4647$ Parkinson's disease and cancer are the most studied pathologies. ${ }^{522}{ }^{24}$ For neurological pathologies, dance is deemed suitable and seems beneficial to adherence, mental health, psychosocial aspects, as well as cognitive, balance and motor skills. ${ }^{22}$ However, no RCTs have been conducted for people after stroke, so the actual effectiveness of dance cannot be determined.

The originality of this RCT is that it evaluates the impact of a dance programme for people with subacute stroke on cognitive and motor recovery, quality of life and the motivation to engage in physical activity. We expect to see the benefits of dance on recovery after stroke, as this activity fosters neuroplasticity ${ }^{14}$ and also entails pleasure and social interaction ${ }^{3}$ - which are factors in regaining motivation. The social component and adaptability of dance could be important motivational factors that favour the improvement of well-being and the fight against sedentariness. ${ }^{9}$

Dance may induce imbalances and risks of falls. But the adverse effects and risks of dance seem very low for persons with neurological conditions. ${ }^{25}$ Dance's safety may be linked to its superior adaptability compared with other sport activities, ${ }^{48}$ and also to the self-regulation of movements by the subjects. Indeed, even if all movements are structured, patients are free regarding their self-imposed constraints. Thus, they are in a situation of self-control regarding their movements and in a stable environment that gives them control over their own safety throughout the activity.

This study presents limitations. Because subjects are recruited in the subacute phase, it is possible that some subjects may have complications that prevent them from completing the programme. Therefore, they will be recruited in a stable medical situation. A limitation could be time and site availability. However, since there is 
already a dance programme in the service's rehabilitation programme, this should not change the centre's general organisation. Blinding is impossible for the subjects and for the dance teacher. Nevertheless, raters are external to the centre and will be unaware of the allocation of subjects between the dance group and the control group.

The results of this study should contribute to new scientific knowledge, providing support for the use of dance in physiotherapeutic approaches as an effective tool for treating persons after stroke.

Contributors EM conceived and designed the study, recruited the participants, drafted and revised the manuscript; JM conceived and designed the study; CJ managed the clinical practice organisation; A-VB-as the study's principal investigator — conceived and designed the study, supervised the study, drafted and revised the manuscript.

Funding This research is funded by an internal grant from the School of Health Sciences, HES-SO//University of Applied Sciences and Arts Western Switzerland, Geneva, Switzerland and a generous patron advised by CARIGEST SA.

Competing interests None declared.

Patient and public involvement Patients and/or the public were not involved in the design, or conduct, or reporting, or dissemination plans of this research.

Patient consent for publication Not required.

Provenance and peer review Not commissioned; externally peer reviewed.

Open access This is an open access article distributed in accordance with the Creative Commons Attribution Non Commercial (CC BY-NC 4.0) license, which permits others to distribute, remix, adapt, build upon this work non-commercially, and license their derivative works on different terms, provided the original work is properly cited, appropriate credit is given, any changes made indicated, and the use is non-commercial. See: http://creativecommons.org/licenses/by-nc/4.0/.

ORCID iD

Anne-Violette Bruyneel http://orcid.org/0000-0003-4764-9336

\section{REFERENCES}

1 López-Ortiz C, Gladden K, Deon L, et al. Dance program for physical rehabilitation and participation in children with cerebral palsy. Arts Health 2012;4:39-54.

2 Sandel SL, Judge JO, Landry N, et al. Dance and movement program improves quality-of-life measures in breast cancer survivors. Cancer Nurs 2005;28:301-9.

3 Quiroga Murcia C, Kreutz G, Clift S, et al. Shall we dance? an exploration of the perceived benefits of dancing on well-being. Arts Health 2010;2:149-63.

4 Green CS, Bavelier D. Exercising your brain: a review of human brain plasticity and training-induced learning. Psychol Aging 2008;23:692-701.

5 Dos Santos Delabary M, Komeroski IG, Monteiro EP, et al. Effects of dance practice on functional mobility, motor symptoms and quality of life in people with Parkinson's disease: a systematic review with meta-analysis. Aging Clin Exp Res 2018;30:727-35

6 Netgen. Organisation de la prise en charge des accidents vasculaires cérébraux en Suisse romande. Revue Médicale Suisse. Available: https://www.revmed.ch/RMS/2017/RMS-N-560/Organisation-de-laprise-en-charge-des-accidents-vasculaires-cerebraux-en-Suisseromande [Accessed 6 Jan 2020].

7 Mahler M-P, Züger K, Kaspar K, et al. A cost analysis of the first year after stroke - early triage and inpatient rehabilitation may reduce long term costs. Swiss Med Wkly 2008;138:459-65.

8 Pollock A, Baer G, Campbell P, et al. Physical rehabilitation approaches for the recovery of function and mobility following stroke. Cochrane Database Syst Rev 2014:CD001920.

9 Brewer L, Horgan F, Hickey A, et al. Stroke rehabilitation: recent advances and future therapies. QJM 2013;106:11-25.

10 Jung J, Lee J, Chung E, et al. The effect of obstacle training in water on static balance of chronic stroke patients. J Phys Ther Sci 2014;26:437-40.

11 Betschart M, McFadyen BJ, Nadeau S. Repeated split-belt treadmill walking improved gait ability in individuals with chronic stroke: a pilot study. Physiother Theory Pract 2018;34:81-90.
12 Oberlin LE, Waiwood AM, Cumming TB, et al. Effects of physical activity on poststroke cognitive function: a meta-analysis of randomized controlled trials. Stroke 2017;48:3093-100.

13 Shariat A, Najafabadi MG, Ansari NN, et al. The effects of cycling with and without functional electrical stimulation on lower limb dysfunction in patients post-stroke: a systematic review with metaanalysis. NeuroRehabilitation 2019;44:389-412.

14 Mang CS, Campbell KL, Ross CJD, et al. Promoting neuroplasticity for motor rehabilitation after stroke: considering the effects of aerobic exercise and genetic variation on brain-derived neurotrophic factor. Phys Ther 2013;93:1707-16.

15 Ploughman M. A review of brain neuroplasticity and implications for the Physiotherapeutic management of stroke. Physiotherapy Canada 2002;54:163-76.

16 Kim GY, Han MR, Lee HG. Effect of Dual-task rehabilitative training on cognitive and motor function of stroke patients. J Phys Ther Sci 2014;26:1-6.

17 Ezeugwu VE, Garga N, Manns PJ. Reducing sedentary behaviour after stroke: perspectives of ambulatory individuals with stroke. Disabil Rehabil 2017;39:2551-8.

18 O'Connell C, Cassidy A, O'Neill D, et al. The aesthetic and cultural pursuits of patients with stroke. J Stroke Cerebrovasc Dis 2013;22:e404-18.

19 Clift SM, Hancox G. The perceived benefits of singing: findings from preliminary surveys of a university College choral Society. J R Soc Promot Health 2001;121:248-56.

20 Patterson KK, Wong JS, Nguyen T-U, et al. A dance program to improve gait and balance in individuals with chronic stroke: a feasibility study. Top Stroke Rehabil 2018;32:1-7.

21 Maglio J, McKinstry C. Occupational therapy and Circus: potential partners in enhancing the health and well-being of today's youth. Aust Occup Ther J 2008:55:287-90.

22 Bruyneel A-V. Effects of dance activities on patients with chronic pathologies: Scoping review. Heliyon 2019;5:e02104.

23 Keogh JWL, Kilding A, Pidgeon P, et al. Physical benefits of dancing for healthy older adults: a review. J Aging Phys Act 2009;17:479-500.

24 Lötzke D, Ostermann T, Büssing A. Argentine tango in Parkinson disease--a systematic review and meta-analysis. BMC Neurol 2015;15:226.

25 Patterson KK, Wong JS, Prout EC, et al. Dance for the rehabilitation of balance and gait in adults with neurological conditions other than Parkinson's disease: a systematic review. Heliyon 2018;4:e00584.

26 Demers M, McKinley P. Feasibility of delivering a dance intervention for subacute stroke in a rehabilitation hospital setting. Int J Environ Res Public Health 2015;12:3120-32.

27 Gowland C, Stratford P, Ward M, et al. Measuring physical impairment and disability with the Chedoke-McMaster stroke assessment. Stroke 1993;24:58-63.

28 Nascimento LR, Caetano LCG, Freitas DCMA, et al. Different instructions during the ten-meter walking test determined significant increases in maximum gait speed in individuals with chronic hemiparesis. Rev Bras Fisioter 2012;16:122-7.

29 Hashimoto $\mathrm{H}$, Takabatake S, Miyaguchi $\mathrm{H}$, et al. Effects of dance on motor functions, cognitive functions, and mental symptoms of Parkinson's disease: a quasi-randomized pilot trial. Complement Ther Med 2015;23:210-9.

30 Shanahan J, Morris ME, Bhriain ON, et al. Is Irish set dancing feasible for people with Parkinson's disease in Ireland? Complement Ther Clin Pract 2015;21:47-51.

31 Winairuk T, Pang MYC, Saengsirisuwan V, et al. Comparison of measurement properties of three shortened versions of the balance evaluation system test (BESTest) in people with subacute stroke. J Rehabil Med 2019;51:683-91.

32 Toglia J, Fitzgerald KA, O'Dell MW, et al. The Mini-Mental state examination and Montreal cognitive assessment in persons with mild subacute stroke: relationship to functional outcome. Arch Phys Med Rehabil 2011;92:792-8.

33 Aguiar LT, Martins JC, Brito SAFde, et al. Knee extensor muscles strength indicates global lower-limb strength in individuals who have suffered a stroke: a cross-sectional study. Braz J Phys Ther 2019;23:221-7.

34 Mentiplay BF, Tan D, Williams G, et al. Assessment of isometric muscle strength and rate of torque development with hand-held dynamometry: test-retest reliability and relationship with gait velocity after stroke. J Biomech 2018;75:171-5.

35 Mentiplay BF, Perraton LG, Bower KJ, et al. Assessment of lower limb muscle strength and power using hand-held and fixed dynamometry: a reliability and validity study. PLOS One 2015;10:e0140822. 
36 de Menezes KKP, Scianni AA, Faria-Fortini I, et al. Measurement properties of the lower extremity motor coordination test in individuals with stroke. J Rehabil Med 2015;47:502-7.

37 Desrosiers J, Rochette A, Corriveau H. Validation of a new lower-extremity motor coordination test. Arch Phys Med Rehabil 2005;86:993-8.

38 Hsueh I-P, Lin J-H, Jeng J-S, et al. Comparison of the psychometric characteristics of the functional independence measure, 5 item Barthel index, and 10 item Barthel index in patients with stroke. $J$ Neurol Neurosurg Psychiatry 2002;73:188-90.

39 Cleary K, Skornyakov E. Predicting falls in community dwelling older adults using the Activities-specific balance confidence scale. Arch Gerontol Geriatr 2017;72:142-5.

40 Laatar R, Kachouri H, Borji R, et al. The effect of cell phone use on postural balance and mobility in older compared to young adults. Physiol Behav 2017;173:293-7.

41 Hou Y-R, Chiu Y-L, Chiang S-L, et al. Development of a smartphonebased balance assessment system for subjects with stroke. Sensors 2019;20:s20010088:88.

42 Legris N, Devilliers H, Daumas A, et al. French validation of the stroke specific quality of life scale (SS-QOL). NeuroRehabilitation 2018;42:17-27.
43 Boiché J, Gourlan M, Trouilloud D, et al. Development and validation of the 'Echelle de Motivation envers l'Activité Physique en contexte de Santé': A motivation scale towards health-oriented physical activity in French. J Health Psychol 2019;24:386-96.

44 Bernhardt J, Hayward KS, Kwakkel G, et al. Agreed definitions and a shared vision for new standards in stroke recovery research: the stroke recovery and rehabilitation roundtable Taskforce. Neurorehabil Neural Repair 2017;31:793-9.

45 Chinsongkram B, Chaikeeree N, Saengsirisuwan V, et al. Reliability and validity of the balance evaluation systems test (BESTest) in people with subacute stroke. Phys Ther 2014;94:1632-43.

46 Hwang PW-N, Braun KL. The effectiveness of dance interventions to improve older adults' health: a systematic literature review. Altern Ther Health Med 2015;21:64-70.

47 Murillo-García Álvaro, Villafaina S, Adsuar JC, et al. Effects of dance on pain in patients with fibromyalgia: a systematic review and meta-analysis. Evid Based Complement Alternat Med 2018;2018:8709748.

48 Fong Yan A, Cobley S, Chan C, et al. The effectiveness of dance interventions on physical health outcomes compared to other forms of physical activity: a systematic review and meta-analysis. Sports Med 2018;48:933-51. 